\title{
Analysis of artificial opals by scanning near field optical microscopy
}

\author{
J. Barrio, ${ }^{1, a)}$ G. Lozano, ${ }^{2, a)}$ J. Lamela, ${ }^{1}$ G. Lifante, ${ }^{1}$ L. A. Dorado, ${ }^{3}$ R. A. Depine, ${ }^{3}$ \\ F. Jaque, ${ }^{1, b)}$ and $\mathrm{H}$. Míguez ${ }^{2, c)}$ \\ ${ }^{1}$ Departamento de Física de Materiales, Universidad Autónoma de Madrid, Cantoblanco 28048 , \\ Madrid, Spain \\ ${ }^{2}$ Instituto de Ciencia de Materiales de Sevilla (CSIC-US), Avenida Américo Vespucio 48, Isla de La Cartuja, \\ 41092 Sevilla, Spain \\ ${ }^{3}$ Departamento de Física, Facultad de Ciencias Exactas y Naturales, Universidad de Buenos Aires, Argentina
}

(Received 16 November 2010; accepted 9 March 2011; published online 20 April 2011)

\begin{abstract}
Herein we present a detailed analysis of the optical response of artificial opal films realized employing a near-field scanning optical microscope in collection and transmission modes. Near-field patterns measured at the rear surface when a plane wave impinges on the front face are presented with the finding that optical intensity maps present a clear correlation with the periodic arrangement of the outer surface. Calculations based on the vector Korringa-Kohn-Rostoker method reproduce the different profiles experimentally observed as well as the response to the polarization of the incident field. These observations constitute the first experimental confirmation of the collective lattice resonances that give rise to the optical response of these three dimensional periodic structures in the high-energy range. (C) 2011 American Institute of Physics. [doi:10.1063/1.3573777]
\end{abstract}

\section{INTRODUCTION}

Since photonic crystals were proposed in $1987,{ }^{1,2}$ they have attracted great interest as they offer potential applications in a wide range of fields. ${ }^{3}$ Three-dimensional photonic crystals have been manufactured in diverse materials and using different procedures ranging from those considered more conventional, such as those based on self-assembly, ${ }^{4}$ to more sophisticated methods, such as those based on direct laser writing $^{5}$ or focus ion beam. ${ }^{6,7}$ Mainly the optical methods used for characterizing these kinds of structures imply the study of both far and near field ranges. Analysis of the far field is adequate for reflectivity studies or stop-gap determinations, ${ }^{8,9}$ whereas near field based techniques allow extracting information on the photon local density of states of these sort of lattices. ${ }^{3}$

The development of the scanning near field optical microscopy (SNOM) has recently opened new possibilities to examining the electric field pattern of one- and twodimensional metallic structures ${ }^{10-12}$ as well as photonic crystals in the near field range. ${ }^{13-21}$ In spite of being widely studied, results for artificial opals, that is, ordered assemblies of monodisperse submicrometric spheres, applying the SNOM technique are very limited at present time. Flück et al. ${ }^{19}$ have reported transmission measurements on polystyrene opals made of 40 monolayers at wavelengths on the order of the lattice parameter in the so-called transmission mode (also known as illumination mode). In this configuration, the photonic crystal is illuminated through an optical fiber with a probe tip aperture of $100 \mathrm{~nm}$, which remains at a constant distance of $<20 \mathrm{~nm}$ from the crystal frontal surface. The transmitted light intensity is collected directly beneath the photonic crystal (PC) by a photodiode detector in contact with the sample.

\footnotetext{
a) These authors contributed equally to this work.

b)Electronic mail: francisco.jaque@uam.es.

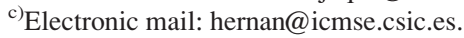

The SNOM images revealed a modulation of the integrated light intensity at the rear surface with the same lattice constant than the frontal illuminated surface. Larger transmission is observed when the tip was positioned at the top of the spheres. More recently, Bittku et al. ${ }^{20}$ reported patterns for eight monolayer thick PMMA opal films grown from $180 \mathrm{~nm}$ radius spheres using the conventional transmission mode. In this case, it was observed that the light transmission intensity map was strongly dependent on intrinsic structural defects along the crystal.

In this paper, SNOM measurements in polystyrene artificial opal thin films made of $0.75 \mu \mathrm{m}$ diameter spheres are compared and reported. The observed transmitted intensity pattern reveals that light is launched more efficiently on top of the spheres rather than between the spheres. These results fairly reproduce and confirm the conclusions reported by Flück et al., ${ }^{19}$ who studied the coupling mechanism of light coming from a pointlike light source to a colloidal crystal. In turn, in collection mode, a quasiplanar wave is used to illuminate the sample, whereas the transmitted light is detected by an optical probe fiber tip located at few nanometers from the spheres in the rear surface. We have found that the SNOM pattern obtained shows a clear periodicity that doubles the photonic crystal lattice constant. Furthermore, this pattern rotates with the polarization of the incident wave, which confirms its relation with the specific type of coupling existing between the incoming wavelength and the lattice. Our results compare well with those attained from simulations performed using a layered vector Korringa-KohnRostoker (KKR) model.

\section{EXPERIMENTAL}

The growth of colloidal crystals from polystyrene (PS) colloids in de-ionized water was carried out using a variation 
of the vertical deposition method developed by Colvin $e t$ al. ${ }^{4}$ For this purpose, commercial PS colloidal particles (IKERLAT) with an average diameter of $0.75 \mu \mathrm{m}$ (polydispersity about $3 \%$ ) were used. Substrates employed were glass microscope slides $(12 \times 76 \mathrm{~mm})$, which were cleaned with doubly distilled water, acetone, carbon tetrachloride, and a 4:1 volume ratio $\mathrm{H}_{2} \mathrm{SO}_{4}: \mathrm{H}_{2} \mathrm{O}_{2}$ solution before being dried with a $\mathrm{N}_{2}$ flow. These substrates were dipped into a cylindrical glass beaker (inner diameter, ca. $25 \mathrm{~mm}$; volume, $25 \mathrm{ml}$ ) containing the PS colloidal suspensions $(15 \mathrm{ml})$ with a concentration of $0.04 \%$ wt. Then the beakers were placed in an oven at $50^{\circ} \mathrm{C}$, and water was evaporated while the colloidal crystal formed on the substrate.

The SNOM images were obtained using a Nanonics Imaging Ltd model MultiView 2000 TM. A continuous wave laser beam from a $532 \mathrm{~nm}$ doubled Nd:YAG laser was coupled into the near-field probe. In the transmission mode, the sample frontal surface is illuminated through the fiber probe with a $\sim 100 \mathrm{~nm}$ tip aperture. The transmitted light is collected through a $10 \times$ long work distance objective and focused into an avalanche photodiode. On the other hand, in the collection mode, the frontal surface of the sample is illuminated directly by the $532 \mathrm{~nm}$ laser beam, and the light transmitted by sample is collected using a $200 \mathrm{~nm}$ tip size fiber coupled to a photon counting system. In the two configurations, the SNOM probe tip works in the tuning fork alternative contact procedure. A data processing program by Nanotec was employed. ${ }^{22}$ It should be remarked that the ratio between the lattice parameter of the artificial opal under analysis and the laser wavelength we use is $a / \lambda=1.98$, which implies that the analysis is being performed for a spectral region in which the field distribution is expected to be strongly influenced by the fine features of the sample.

\section{RESULTS AND DISCUSSION}

Figure 1 shows schematic drawings of the experimental setups used for our analysis. To perform a measurement in transmission mode [see Fig. 1(a)], the sample is illuminated through an optical fiber probe with subwavelength aperture. Such probe is located very close to the colloidal crystal outer surface. Light is detected through a long working distance
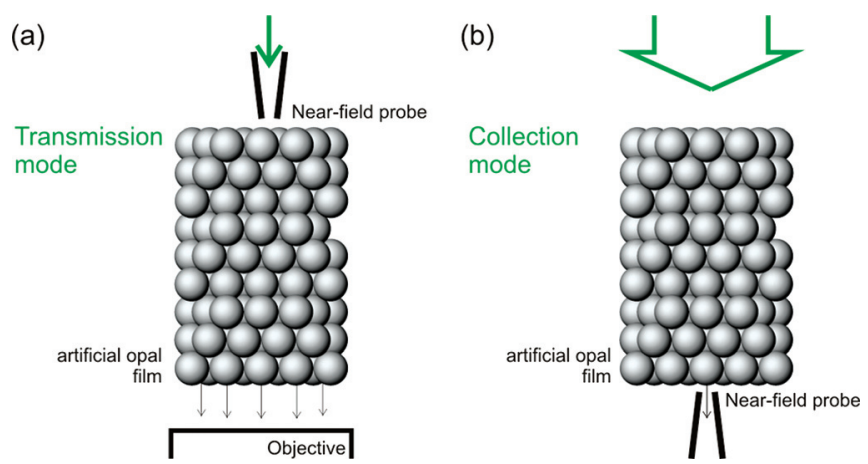

FIG. 1. (Color online) Schematic drawings of the near-field setups used for our analysis. (a) In the transmission mode, light is launched from a near-field probe to the artificial opal film. An objective beneath the sample detects all the light reaching the rear side of the sample. (b) In the collection mode, a quasiplanar wave is used to illuminate the slab, whereas the transmitted light through the sample is collected by an optical probe located close to the spheres. objective placed beneath the sample. For this reason, the topographic and the optical information obtained by using this mode do not correspond to the same surface of the colloidal crystal: light impinges on one side of the crystal under near optical field conditions, whereas the detector records the integrated light intensity that exits the sample on the opposite side. On the other hand, in the collection mode [see Fig. 1(b)], a planar wave illuminates a relatively large area (on the order of several square micrometers) of the front face of the colloidal crystal film, and an optical fiber tip, located at a few nanometers from the spheres, is employed to collect the light transmitted through the rear side. In this latter case, both topographic and optical information obtained correspond to the same face and local area of the sample.

\section{A. Transmission mode}

Figures 2(a) and 2(b) show, respectively, the topographic and the optical images obtained by using a SNOM in the transmission mode. In Fig. 2(a), we show the well known triangular arrangement of the spheres corresponding to the outer surface of the photonic crystal, which corresponds to the (111) plane of a slightly distorted face-center-cubic structure. ${ }^{23}$ It is important to point out that no information about the spatial distribution of the transmitted near-field could be extracted from Fig. 2(b) because the objective harvests all light exiting from the bottom crystal facet without spatial discrimination. Instead, this mode provides information on the efficiency of energy transfer as a function of the position of the illuminating tip at the frontal surface. Figures 2(c) and 2(e) show, respectively, the two-dimensional (2D) self-correlation (SC) and the fast Fourier transform (FFT) analysis of the topographic image. In the FFT representation, the $k=0$ and the first-order reciprocal-lattice are observed. The sharpness of these spots indicates that the lattice is well defined. The SC image supports these assumptions. It is important to mention here that the crystal surface shows imperfections such as dislocations, vacancies, or cracks. This way, the SC is employed as a tool for finding a repeating pattern. In our case, this function allows revealing a periodic signal, related to the ordered structure, which lay hidden under the
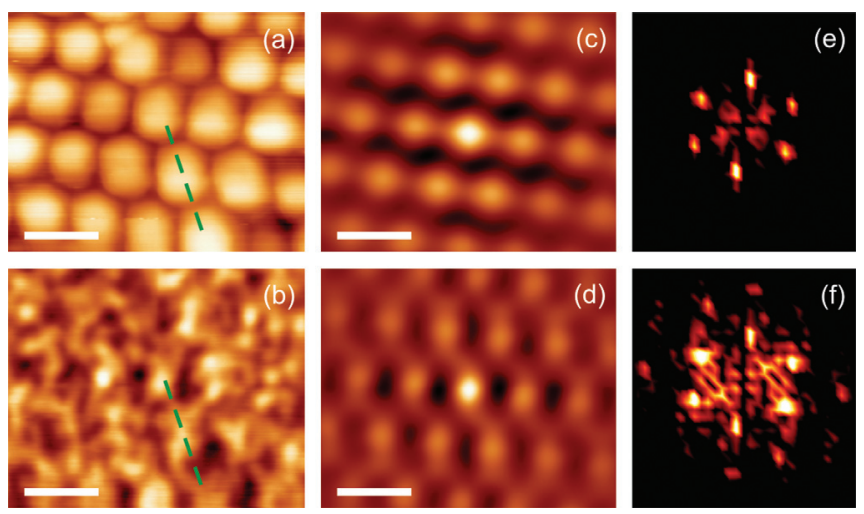

FIG. 2. (Color online) (a) 2D topographic image of the illuminated surface of the PC. (b) 2D light intensity distribution using the SNOM transmission mode. Self-correlation analysis of the topographic (c) and the optical (d) images presented in (a) and (b), respectively. Scale bars are $1 \mu \mathrm{m}$. 2D Fourier Transforms of the topographic (e) and the optical (f) images presented in (a) and (b), respectively. 


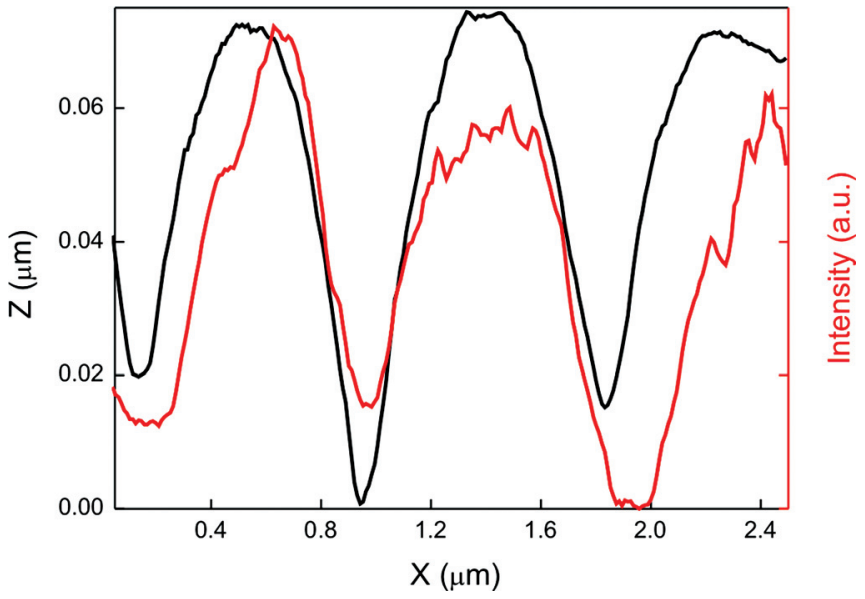

FIG. 3. (Color online) Topographic (black) and optical (red/grey) profiles obtained along the line traced in Figs. 2(a) and 2(b), respectively.

unavoidable noise caused by the presence of intrinsic defects However, despite these anomalies, the triangular arrangement mentioned in the preceding text is preserved. As a working protocol, zones away from leaps between terraces of different thickness ${ }^{24}$ or domain boundaries were always chosen.

At first glance, the optical image does not clearly show a periodicity in the transmitted light intensity as it can be seen in Fig. 2(b). However both the SC and FFT images displayed in Figs. 2(d) and Fig. 2(f), respectively, reveal a modulation of the spatially integrated transmitted light intensity with the same lattice constant of the frontal surface of the colloidal crystal, a hexagonal pattern of bright intense spots being clearly visible. Topographic and optical profiles along the line traced in Figs. 2(a) and 2(b) are plotted in Fig. 3 as black and red lines, respectively. In all the measurements, more transmitted light is detected when the illuminating tip is positioned on the top of a sphere, and less light is collected when the near-field probe is placed between the spheres. The results of our optical analysis confirm the data previously reported by Flück et al. ${ }^{19}$ As they claimed, optimized coupling between incoming radiation and the (111) surface of an artificial opal is achieved when light is launched at the top of a sphere.

\section{B. Collection mode}

In this mode, a quasiplanar wave is used to illuminate the frontal surface of the colloidal crystal, whereas the transmitted light is detected by an optical probe located at a few nanometers from the spheres in the rear surface. Notice that this is the same illumination configuration employed when standard far optical field measurements, such as reflectance or transmittance, are performed. Therefore this setup could allow observing the fine features of the transmitted optical field before reconstruction takes place away from the sample to yield the well-known homogeneous distribution of the farfield. In Fig. 4, we present the transfer of light transmitted through the sample and collected by a near-field fiber probe. Figure 4(a) shows the topographical information obtained simultaneously with the near-field pattern presented in Fig. 4(b). The comparison of these two images allows us analyzing the spatial distribution of the transmitted near-field when
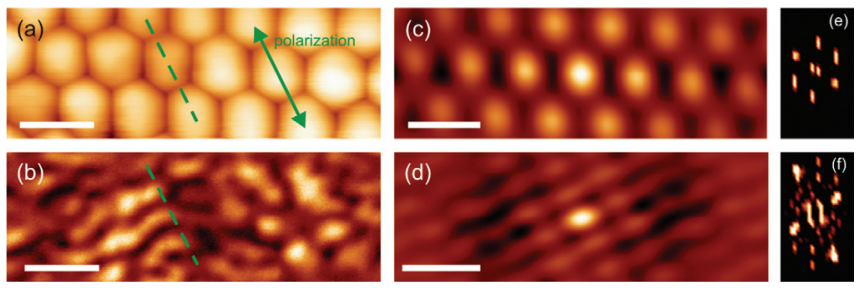

FIG. 4. (Color online) (a) 2D Topographic image of the outer surface of the PC. (b) 2D-light intensity distribution using the SNOM collection mode. Self-correlation analysis of the topographic (c) and the optical (d) images presented in (a) and (b), respectively. Scale bars are $1 \mu \mathrm{m}$. 2D Fourier transforms of the topographic (e) and the optical (f) images presented in (a) and (b), respectively. The polarization direction is indicated in the AFM image.

a planar wave impinges on the artificial opal film. As is expected, the AFM image presented in Fig. 4(a) reveals the same triangular arrangement observed in Fig. 2(a). Interestingly, the 2D FFT and the SC of the optical image [see Figs. 4(d) and 4(f)] exhibit a diffraction pattern similar to the one displayed in Fig. 4(e). This fact indicates that the light intensity distribution throughout the area analyzed is clearly related to the periodic structure. As far as we know, this is the first time a periodic profile of the near field pattern is observed from an artificial opal by using the same illumination configuration employed when standard far optical field measurements are performed.

Figure 5 shows the topographic and optical profiles recorded along the line traced in Figs. 4(a) and 4(b). The periodicity of the topographic pattern corresponds to the arrangement of the polystyrene spheres, which is $0.75 \mu \mathrm{m}$. The FFT and SC analysis of the optical profile reveals a clear periodic modulation of the light intensity, consisting of two maxima and one minimum centered at the position in which each sphere is placed. The same profile structure has been found along different rows of spheres presenting the same relative orientation with respect to the incident electric field. However, the relative peak intensity varies sensibly along the sample which is probably related to the presence of intrinsic defects. This issue makes difficult the direct observation of a well defined periodic profile when large areas of the crystal are scanned. The observation of the effect of rotating the polarization of the incident wave on the

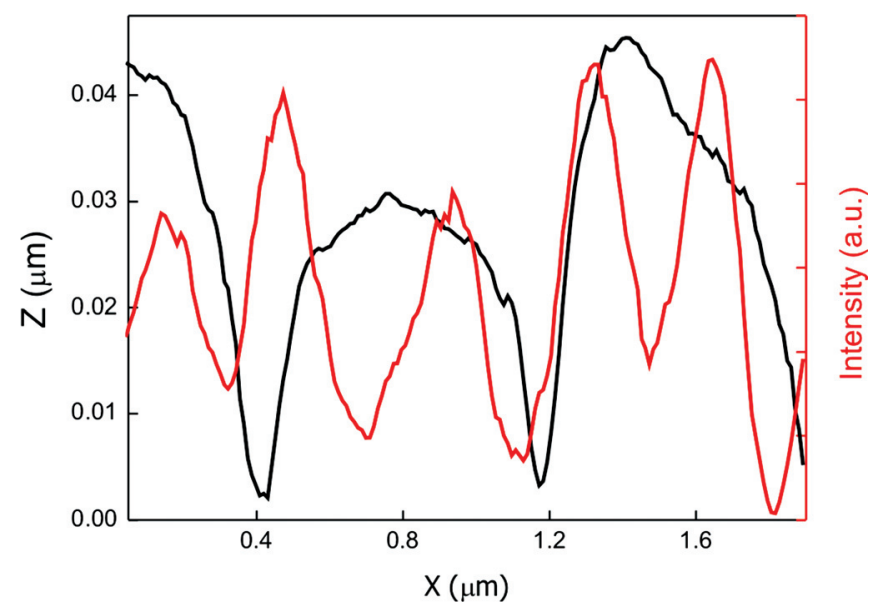

FIG. 5. (Color online) Topographic (black) and optical (red/grey) profiles obtained along the line traced in Figs. 4(a) and 4(b), respectively. 

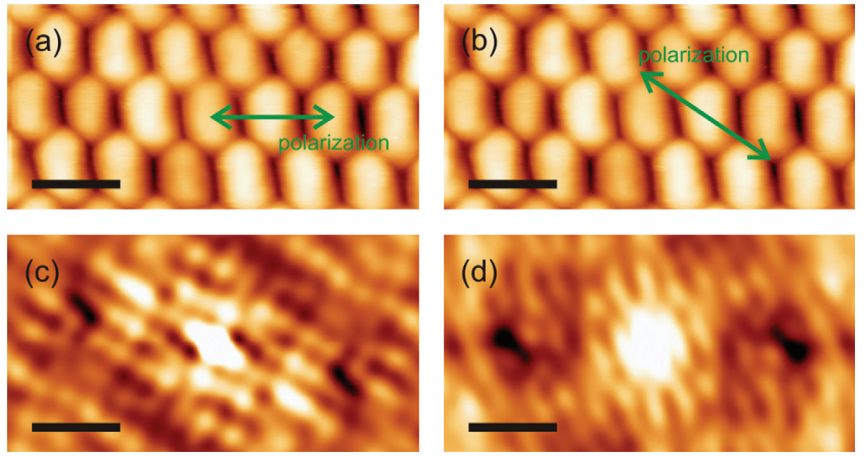

FIG. 6. (Color online) Topographic images of a selected region of the rear surface of an artificial opal, (a) and (b). SNOM images taken when the incident electric field is linearly polarized along a row of spheres (c) and after rotating the polarization direction $30^{\circ}(\mathrm{d})$. The polarization directions are indicated with arrows in the AFM images. Scale bars are $1 \mu \mathrm{m}$.

near-field pattern further confirms that the lattice periodicity modulates the near-field intensity profile. Although a detailed description of the polarization dependence of the SNOM patterns is beyond the scope of this study, we could confirm that the periodic light intensity pattern measured rotates with the polarization of the incident wave, as displayed in Fig. 6.

\section{Numerical simulations}

Interpretation of near-field data is complicated, and the support of a simulation model is generally helpful to contrast the experimental data. To provide some insight into the physical origin of the periodic light collected intensity pattern and the corresponding polarization dependence, we use a vector KKR based method, which has been recently employed to accurately analyze the spectral optical response of artificial opals in the high energy range. ${ }^{25}$ The physical mechanisms that describe the fine features of the transmitted optical field based on collective properties of the lattice dynamics, each spectral feature having its origin in the resonance of a specific mode inside the crystal slab, which resonates as a whole entity due to electromagnetic links between the lattice components. Prior to this work, we have already shown that all optical spectral features in the high energy region of photonic crystals arise from electromagnetic resonances within the ordered array, modified by the interplay between these resonances with the opening of diffraction channels, the presence of imperfections, and the finite size effects. ${ }^{23,25}$ Most importantly, the periodic modulation of the intensity of the near-field pattern herein reported constitutes the first experimental evidence of the direct observation of such resonances.

In Fig. 7(a), we show the calculated transmittance spectrum of a 10-layer colloidal crystal film in reduced units of a/ $\lambda$. In all our experiments $\lambda=532$. This value corresponds to the high-energy range $(\mathrm{a} / \lambda=1.98)$; it is well above the predicted cut-off at $\mathrm{a} / \lambda=1.63$ for diffracted beams in air and is indicated by a vertical dashed line in Fig. 7(a). In Figs. 7(b) and 7(c), we show the spatial dependence of the calculated transmitted electric field $\left(\vec{E}_{\mathrm{t}}\right)$ when an incident plane wave propagates through a glass supported colloidal crystal. In Figs $7(\mathrm{~b})$ and $7(\mathrm{c})$, we plot the $\left|\vec{E}_{\mathrm{t}}(x, y, z)\right|$ calculated in the
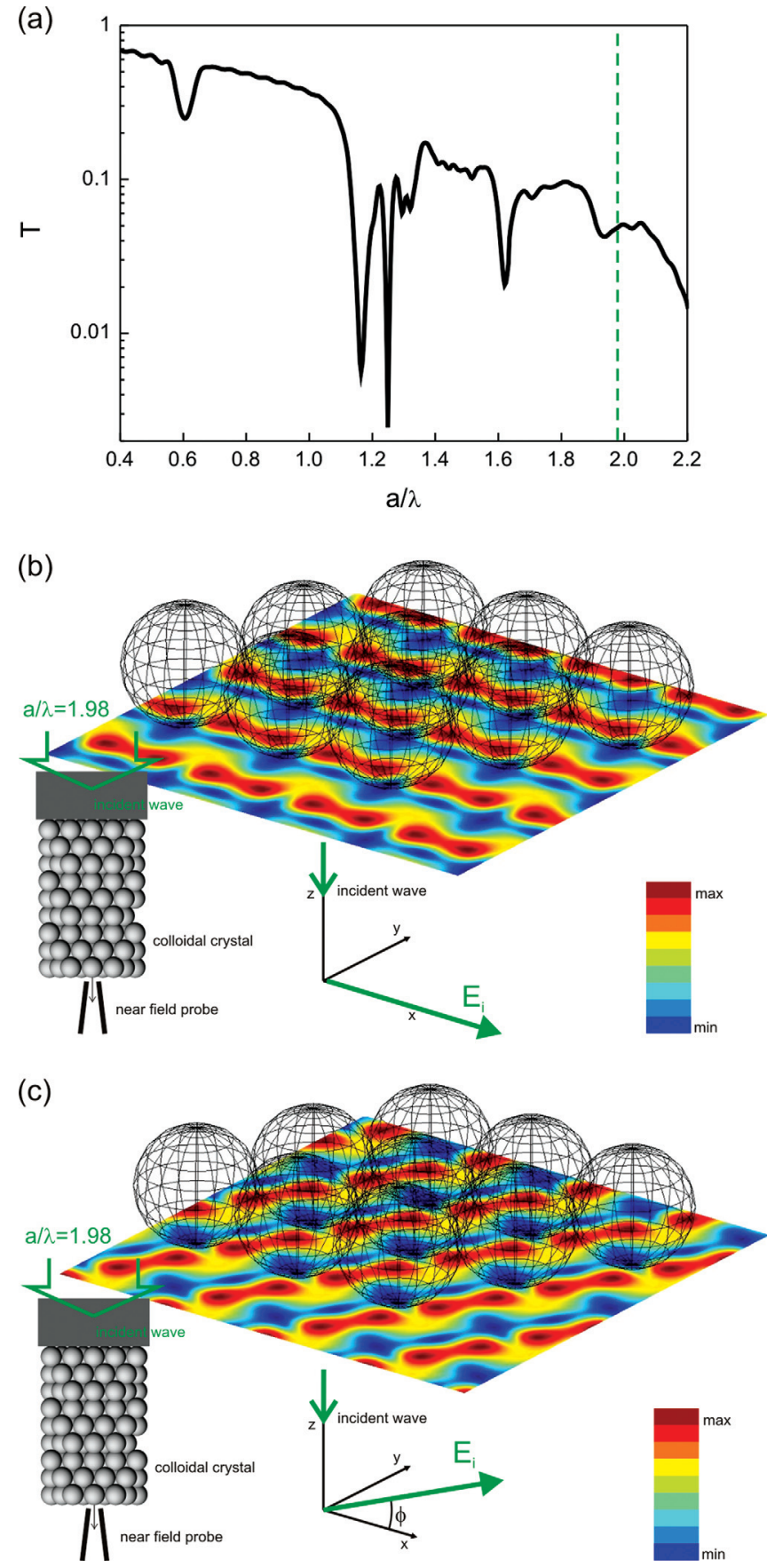

FIG. 7. (Color online) (a) Calculated normal incidence transmittance spectrum for a 10-layer glass supported colloidal crystal made of spheres of dielectric constant $\varepsilon_{s}=2.5+0.05 i$. Vertical dashed line indicates the experimental incident wavelength value $(\lambda=532)$. (b) and (c) Spatial distribution of the intensity of the transmitted electric field calculated in the plane tangent to the poles of the spheres that form the outer compact plane in the 10layer stack. The angle $\phi$ that forms the incident electric field respect to the normal of the outer surface of the colloidal crystal ( $z$ axis in our reference system) is $\phi=0^{\circ}$ in (b) and $\phi=60^{\circ}$ in (c). Color (intensity) scales are also indicated.

plane tangent to the poles of the spheres that form the outer compact plane in the 10-layer stack for two different values of the angle $\phi$ that forms the incident electric field with the $x$ axis in our reference system, specifically $\phi=0^{\circ}$ in (b) and $\phi=60^{\circ}$ in (c). Our results indicate on the one hand that the light intensity distribution throughout this outer plane is related to the periodic structure and in the other hand that the 
periodic light intensity pattern rotates with the polarization of the incident wave, which is in excellent agreement with our measurements.

As long as the physical origin of the optical response of an artificial opal lies on multipolar resonances of the sphere ensemble, the coupling of light from or to an artificial opal depends on the relative launching position with respect to the crystal lattice and the collective properties of the lattice dynamics. This way, the observation of the periodic profile of the near field intensity herein reported represents the first experimental and theoretical confirmation of the resonances that result in the optical response of an artificial opal.

\section{CONCLUSIONS}

We have studied the optical patterns obtained from artificial opals when analyzed using a near-field scanning optical microscope. The analysis of the optical pattern obtained in transmission configuration reveals that light is launched more efficiently on top of the spheres rather than between the spheres, confirming previous results obtained by Flück et al. Optical images attained working in collection mode show a clear correlation with the topographic information simultaneously measured from the colloidal crystal. Light intensity profiles consist of a double peak structure with its minimum located at the top of each sphere. Furthermore, this pattern rotates with the polarization of the incident wave. We have also performed KKR vector method based calculations to reproduce the optical images attained in fair agreement with the measurements performed being found.

\section{ACKNOWLEDGMENTS}

This work has been partially supported by Comunidad Autónoma de Madrid (Spain) under the project MICROSERES (Ref. S2009/TIC-1476), the Spanish Ministry of Science and Innovation under Grant MAT2007-02166 and Consolider HOPE CSD2007-00007 and Junta de Andalucía under Grants FQM3579 and FQM5247. G.L. thanks CSIC for funding through an I3P scholarship.
${ }^{1}$ E. Yablonovitch, Phys. Rev. Lett. 58, 2059 (1987).

${ }^{2}$ S. John, Phys. Rev. Lett. 58, 2486 (1987).

${ }^{3}$ K. Sakoda, Optical Properties of Photonics Crystals, Springer Series in Optical Sciences, Vol. 80 (Springer, New York, 2001).

${ }^{4}$ P. Jiang, J. F. Bertone, K. S. Hwang, and V. L. Colvin, Chem. Mater. 11, 2132 (1999).

${ }^{5}$ M. Deubel, G. Von Freymann, M. Wegener, S. Pereira, K. Busch, and C. M. Soukoulis, Nat. Mater. 3, 444 (2004).

${ }^{6}$ Photonic Crystals: Advances in Design, Fabrication and Characterization, edited by K. Busch, S. Lölkes, R. B. Wehrspohn, and H. Föll (Wiley, VCH, Berlin, 2004).

${ }^{7}$ F. Lacour, N. Courjal, M. P. Bernal, A. Sabac, C. Bernier, and M. Spajer, Opt. Mater. 27, 1421 (2005).

${ }^{8}$ L. A. Dorado, R. A. Depine, D. Schinca, G. Lozano, and H. Míguez, Phys. Rev. B 78, 075102 (2008).

${ }^{9}$ J. F. Galisteo-López and C. López, Phys. Rev. B 70, 035108 (2004).

${ }^{10}$ C. Ropers, D. J. Park, G. Stibenz, G. Steinmeyer, J. Kim, D. S. Kim, and C. Lienau, Phys. Rev. Lett. 94, 113901 (2005).

${ }^{11}$ T. Rindzevicius, Y. Alaverdyan, B. Sepulveda, T. Pakizeh, M. Käll, R. Hillenbrand, J. Aizpurua, and F. J. García de Abajo, J. Phys. Chem. C 111, 1207 (2007).

${ }^{12}$ J. A. Schuller, E S. Barnard, W. Cai, Y. C. Jun, J. S. Whiteand, and M. L. Brongersma, Nat. Mater. 9, 193 (2010).

${ }^{13}$ P. L. Phillips, J. C. Knight, B. J. Mangan, P. St. J. Russell, M. D. B. Charlton, and G. J. Parker, J. Appl. Phys. 85, 6337 (1999).

${ }^{14}$ M. L. M. Balistreri, H. Gersen, J. P. Korterik, L. Kuipers, and N. F. van Hulst, Science 294, 1080 (2001).

${ }^{15}$ S. I. Bozhevolnyi, V. S. Volkov, T. Søndergaard, A. Boltasseva, P. I. Borel, and M. Kristensen, Phys. Rev. B 66, 235204 (2002).

${ }^{16}$ H. Gersen, J. P. Korterik, N. F. van Hulst, and L. Kuipers, Phys. Rev. E 68, 026604 (2003).

${ }^{17}$ E. Flück, M. Hammer, A. M. Otter, J. P. Korterik, L. Kuipers, and N. F. van Hulst, J. Light Tech. 21, 1384 (2003).

${ }^{18}$ H. Gersen, T. J. Karle, R. J. P. Engelen, W. Bogaerts, J. P. Korterik, N. F. van Hulst, T. F. Krauss, and L. Kuipers, Phys. Rev. Lett. 94, 073903 (2005).

${ }^{19}$ E. Flück, N. F. van Hulst, W. L. Vos, and L. Kuipers, Phys. Rev. E 68, 015601 (2003).

${ }^{20}$ K. Bittkau, R. Carius, A. Bielawny, and R. B. Wehrspohn, J. Mater. Sci.: Mater. Electron. 19, 203 (2008).

${ }^{21}$ S. Vignolini, M. Burresi, S. Gottardo, L. Kuipers, and D. S. Wiersma, Opt. Lett. 35, 2001 (2010).

${ }^{22}$ I. Horcas, R. Fernández, J. M. Gómez-Rodríguez, J. Colchero, J. Gómez-Herreros, and A. M. Baro, Rev. Sci. Instrum. 78, 013705 (2007).

${ }^{23}$ G. Lozano, L. A. Dorado, D. Schinca, R. A. Depine, and H. Míguez, Langmuir 25, 12860 (2009).

${ }^{24}$ G. Lozano and H. Míguez, Langmuir 23, 9933 (2007).

${ }^{25}$ L. A. Dorado, G. Lozano, H. Míguez, and R. A. Depine, Phys. Rev. B 76, 245103 (2007). 